\title{
REACA: An Efficient Protocol Architecture for Large Scale Sensor Networks
}

\author{
Zhi Quan, Ananth Subramanian, Member, IEEE, and Ali H. Sayed, Fellow, IEEE
}

\begin{abstract}
The emergence of wireless sensor networks has imposed many challenges on network design such as severe energy constraints, limited bandwidth and computing capabilities. This kind of networks necessitates network protocol architectures that are robust, energy-efficient, scalable, and easy for deployment. This paper proposes a robust energy-aware clustering architecture (REACA) for large-scale wireless sensor networks. We analyze the performance of the REACA network in terms of quality-of-service, asymptotic throughput capacity, and power consumption. In particular, we study how the throughput capacity scales with the number of nodes and the number of clusters. We show that by exploiting traffic locality, clustering can achieve performance improvement both in capacity and in power consumption over general-purpose ad hoc networks. We also explore the fundamental trade-off between throughput capacity and power consumption for single-hop and multihop routing schemes in cluster-based networks. The protocol architecture and performance analysis developed in this paper provide useful insights for practical design and deployment of large-scale wireless sensor network.
\end{abstract}

Index Terms-Clustering, multi-hop routing, performance analysis, throughput capacity, wireless sensor networks.

\section{INTRODUCTION}

$\mathbf{W}$ IRELESS sensor networks (WSNs) consist of spatially distributed sensor devices with sensing, wireless communications, and computation capabilities. These wireless networks have broad applications, e.g., environment monitoring, target tracking, and surveillance. Unlike mobile ad hoc networks (MANETs), WSNs are usually application-specific. The unique characteristics of WSNs such as limited bandwidth and computing capacity, and severe energy constraints, make their design more challenging. One essential issue in the design of WSNs is how to use bandwidth and energy resources efficiently while prolonging the system lifetime. In this paper, we examine the following important design considerations.

\section{A. Throughput Capacity}

Since WSNs may consist of a large number of sensor nodes, protocols should provide good throughput capacity as the number of sensor nodes increases. In recent work [1], it has been shown that the per node throughput capacity of a general-purpose non-clustered wireless network ${ }^{1}$ is

Manuscript received December 8, 2005; revised April 3, 2006; accepted September 6, 2006. The associate editor coordinating the review of this paper and approving it for publication was H.-H. Chen. This work was partially supported by NSF grants ECS-0401188 and ECS-0601266.

Z. Quan and A. H. Sayed are with the Department of Electrical Engineering, University of California, Los Angeles, CA 90095-1594 USA (e-mail: quan@ee.ucla.edu; sayed@ee.ucla.edu).

A. Subramanian is with the Institute for Infocomm Research, A-STAR, Singapore (e-mail: msananth@ieee.org).

Digital Object Identifier 10.1109/TWC.2007.05964.

${ }^{1}$ The notation $y=\Theta(f(N))$ is used to signify that there exist positive constants $\kappa_{1}$ and $\kappa_{2}$ such that $\kappa_{1} f(N) \leq y \leq \kappa_{2} f(N)$.
$\Theta\left(R / \sqrt{N_{t} \log N_{t}}\right)$, where $R$ is the common transmission rate of each node and $N_{t}$ is the total number of nodes in the network. The result implies that the per node throughput capacity approaches zero as the network size increases. Therefore, it is preferable to cluster together nodes that are geographically close and mostly communicate with each other.

\section{B. Energy Conservation and Awareness}

Moreover, since the sensor nodes are battery operated, energy conservation is extremely important. In order to maximize the system lifetime, protocols should alleviate the hot spot problem in routing and evenly distribute the energy load among all the nodes, so that there are no overly-used nodes that will run out of energy before the others.

\section{Robustness}

Still, it is quite possible that some nodes will fail or be blocked due to lack of energy, physical damage, or environmental interference. In the proposed protocol, the failure of some sensor nodes will not prevent the entire network from operating.

In this paper, we develop a robust energy-aware clustering architecture (REACA) to meet the design requirements of WSNs. This architecture supports data aggregation and enables access to information of interest from data collected by spatially distributed sensor nodes. Applications include the average temperature of a field, an anomaly in a surveillance network, and the location of a particular event, etc. We analyze the performance of the REACA network in terms of quality-ofservice (blocking probability), throughput capacity, and power consumption, and show how these performance measures scale with the size of the network. Our results show that if the number of clusters is medium or large, the REACA network can achieve better performance than a general-purpose ad hoc wireless network.

This paper is organized as follows. In Section II, we review some related work. The REACA structure is described in Section III. Throughout Sections IV-VI, we present the performance analysis. Section VII concludes this paper with discussion on some future research.

\section{RELATED WORK}

The development of low-energy protocols for WSNs has attracted attention in recent years [2] [3]. It has been shown in [4], for instance, that clustering enables bandwidth reuse and scalability, and thus improves system capacity. In [5], a linked cluster architecture was proposed and in [6] three effective solutions for joint clustering and power control are described. 
Moreover, in [7], a joint rate and power control scheme is presented that achieves a desired level of signal-to-interference and noise ratio (SINR) at the cluster head node.

Likewise, energy-aware routing protocols [8]-[10] have been developed to prolong the network lifetime. The basic strategy of these protocols is to select routes based on the energy at each node on the route. Recently, a low-energy adaptive clustering hierarchy (LEACH) protocol for applicationspecific WSNs was developed in [11]. LEACH achieves this goal by randomly selecting a few sensor nodes as cluster heads so as to evenly distribute the energy load among nodes in the entire network. Although LEACH can achieve a long system lifetime, the dynamic clustering brings in protocol overhead and may result in isolated nodes. Also, LEACH requires that transmission within the cluster be completed through a single hop, which is not energy efficient if the two nodes are located far away from each other.

In this paper, we develop an alternative architecture (REACA) to address these issues. This protocol architecture differs from LEACH in the following aspects. First, REACA uses a predetermined clustered structure and applies energyawareness by selecting a node with most energy as the master node (or cluster head) for each cluster. This circumvents the unnecessary protocol overhead and possible isolated nodes in the network. Second, to achieve energy efficiency, REACA adopts multi-hop routing when the source and destination nodes are far apart. In a sense, REACA may be considered as a generalization of the LEACH protocol. More importantly, this paper provides asymptotic analysis for scaling network performance in terms of the blocking probability, capacity, and power consumption.

\section{REACA NETWORK}

We consider a space covered by $M$ predetermined geographical clusters ${ }^{2}$ as shown in Fig. 1. Each cluster contains $N$ terminal nodes and one master node, and thus, the total number of nodes in the network is given by $N_{t}=(N+1) \times M$. A master node performs important tasks, such as necessary signal processing, sending data packets to a base station (BS), and networking information management. To maximize the network lifetime, we let each node of a cluster take its turn to serve as the master. Although the master node is more powerintensive than terminal nodes, the fraction of time it functions as a master node is only about $1 /(N+1)$.

The protocol operation is divided into cycles [12]. As shown in Fig. 2, each cycle begins with a setup phase when the master nodes are determined for each cluster. Following the setup phase is the transmission phase, during which the terminal nodes send data to their own master nodes.

\section{A. Master Selection}

The strategy of energy-awareness is applied to maximize the system lifetime. During the setup phase, candidates of the master node broadcast a message through an exclusive

\footnotetext{
${ }^{2}$ The formation of clusters depends on specific applications. For energy and bandwidth efficiency, we cluster nodes that are close to and have strong correlation with each other. Other clustering approaches appear in [4], [6], [11] and the references therein.
}

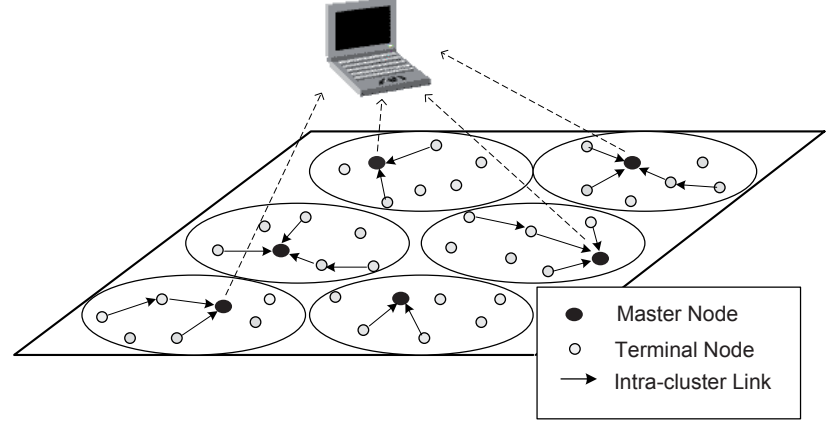

Fig. 1. A generic model for REACA networks.

\begin{tabular}{|c|c|c|c|}
\hline $\begin{array}{l}\text { Setup } \\
\text { Phase }\end{array}$ & Transmission Phase & $\begin{array}{l}\text { Setup } \\
\text { Phase }\end{array}$ & Transmission Phase \\
\hline Ts & $\mathrm{Tt}$ & Ts & $\mathrm{Tt}$ \\
\hline
\end{tabular}

Fig. 2. The protocol operation shown in a time scale. Once a master node is chosen during the setup phase, packets are transmitted from terminal nodes to the master node during the transmission phase. In general, the duration of transmission phases is much longer than that of setup phases, i.e., $T_{t} \gg T_{s}$.

frequency channel using the CSMA/CA protocol. The message indicates the available energy and the node's unique identifier. Therefore, each node can automatically determine the master node without a centralized decision maker.

\section{B. The Routes of Packets}

In WSNs with data aggregation capability, the sensor nodes send processed information instead of original observations to the base station or fusion center. A simple example of such an application is a WSN that measures the average temperature of a field. In this application, each node individually measures its surrounding temperature and sends the measurement to the master node. The master node takes the average of measurements over all nodes within the cluster and over a period of time, i.e., $T_{m}$ cycles, and then sends the result to the base station for further processing.

Without loss of generality, the intra-cluster traffic amounts to the majority of the overall network traffic. According to the available energy on each node, routes of packets are established either through a single hop or through multiple hops via relay nodes within the cluster. The single-hop transmission is similar to $\mathrm{LEACH}$, in which the terminal node sends data packets directly to the destination node. Nevertheless, WSNs are usually energy constrained and prefer multi-hop routing. To secure efficiency, the routes should be over nearly straightline paths. An example is illustrated in Fig. 3. At time cycle $t$, packets generated from the source node are forwarded to the master node if the master node is in its route to the final destination; otherwise, the packets wait in the buffer until the master node appears in any one of the possible routes. At time cycle $t+1$, the previous master node transfers the relayed packets together with its own generated traffic to the current master node if the current master node is in the route to the final destination. If the destination node becomes the 


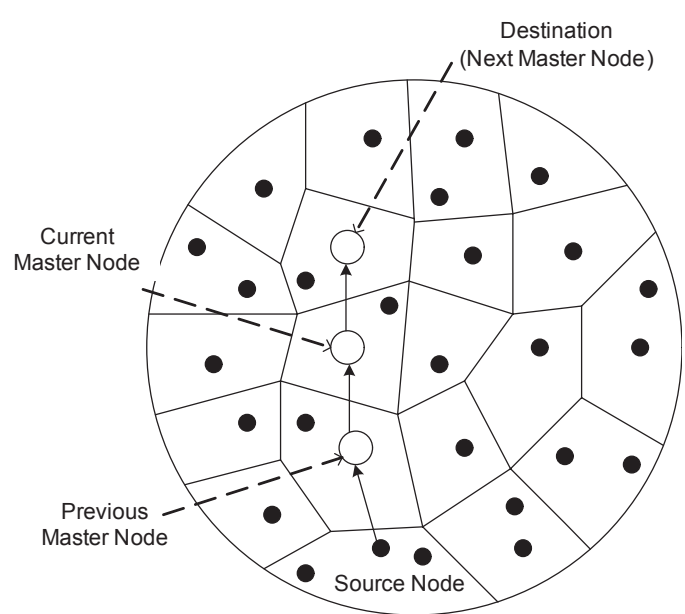

Fig. 3. Example of a multi-hop route within the cluster. The cluster is divided into many cells, each of them contains at least one node.

master at time $t+3$, the packets will then be forwarded to the destination. Thus, a successful multi-hop transmission may take many cycles.

The master nodes process information collected from the terminal nodes and then send the result to the BS. The communication between the master and the BS uses a different channel from intra-cluster communications, and this amount of traffic will be negligible compared with the intra-cluster traffic when $T_{m}$ is large. Above and throughout this paper, we assume that each node has enough power to transmit data packets to the BS and has a large buffer for relayed traffic.

\section{Transmission Model}

Consider $N+1$ nodes independently and uniformly distributed in a cluster $\mathcal{A}$ with an area of $A$. When node $i$ communicates with its master node, it suffers interference from the nodes in other clusters using the same channel and from background noise. Let $\mathcal{T}$ be the set of nodes from different clusters simultaneously transmitting over the channel. At time $t$, the transmission from a terminal node of cluster $i$ can be successfully received by its master node if the SINR at the master node satisfies

$$
\frac{P_{i}(t) G_{i i}(t)}{\sum_{j \in \mathcal{T}, j \neq i} P_{j}(t) G_{j i}(t)+\sigma_{i}^{2}} \geq \gamma
$$

where $G_{j i}$ denotes the channel gain from the $j$-th transmitting node to the master node of cluster $i, \sigma_{i}^{2}$ is thermal noise at the receiver of the master node of cluster $i, P_{i}$ is the transmission power of node $i$, and $\gamma$ is a certain threshold.

In a fading and shadowing channel, the channel gain is modelled as

$$
G_{j i}=S_{0} \frac{10^{\eta / 10}}{d_{j i}^{\beta}}
$$

where $d_{j i}$ denotes the distance between the nodes, $S_{0}$ is a function of the carrier frequency, $\beta$ denotes the path loss exponent, and $\eta$ is a zero mean Gaussian random variable with variance $\sigma_{\eta}^{2}$ (i.e., $10^{\eta / 10}$ represents the shadowing factor with a lognormal distribution). In practice, the values of $\beta$ and $\sigma_{\eta}$ depend on the physical environment and usually have $2<\beta<6$ and $6<\sigma_{\eta}<12$.

\section{Media Access Control (MAC)}

For specific applications, the cluster needs several simultaneous transmissions in each cycle. We adopt time division multiple access (TDMA) to transmit packets for bandwidth and energy-efficiency considerations. The master node divides the transmission time into $Q$ time slots and selects at most $Q$ terminal nodes to transmit packets. This scheduling information is sent to nodes in the cluster such that no collision will happen among transmitting nodes and the non-transmitting nodes can switch to a sleeping state for energy conservation.

Throughout this paper, the analysis is carried out based on an asymptotic regime, i.e., with a probability approaching one as $N \rightarrow \infty$, denoted by "with high probability (w.h.p.)".

\section{Blocking Probability}

With the protocol described above, we define the blocking probability in a cluster as $\operatorname{Pr}\left(Z_{N}>Q\right)$, where $Z_{N}$ is the number of nodes that simultaneously express a desire to connect to the master node. In this section, we will study the blocking probability in two scenarios: $\alpha$-prioritized networks [13] and distance-based probabilistic connections.

\section{A. $\alpha$-Prioritized Networks}

Assuming that no two nodes have the same probability to connect to the master node at any time, the definition of the $\alpha$-prioritized network is given as follows.

Definition 1 (Ordered Chain): An ordered chain is a set of real numbers where its $i$-th element is less than its $j$-th element if $i>j$ (i.e., it is a set of strictly decreasing real numbers).

Definition 2 (Prioritized Cluster): A prioritized cluster is a cluster in which the set of all probabilities that a terminalmaster pair becomes active forms an ordered chain.

Definition 3 ( $\alpha$-Prioritized Cluster): An $\alpha$-prioritized cluster is a prioritized cluster in which the set of all probabilities that a terminal-master node pair becomes active is uniformly bounded above by a geometric series $\left\{\alpha^{k}, k \geq 0\right\}$ for some known and fixed real number, $0<\alpha<1$.

The $\alpha$-prioritized networks form a wide class of networks considering the fact that many networks have nodes that are widely and uniformly distributed and with a minimum distance between any two nodes. The following result gives an expression for how $Q$ should scale with $N$ if we desire to maintain a fixed bound on the blocking probability, say $1 / \nu$, for any $N$.

Proposition 1 (Time Slots and Blocking Probability): For an $\alpha$-prioritized cluster with a master node and $N$ terminal nodes, the number of time slots sufficient to ensure a maximum blocking probability of $1 / \nu$ (with $\nu \geq 1$ ) is

$$
Q=\sqrt{2 N \ln \nu}+\frac{1-\alpha^{N+1}}{1-\alpha}
$$

This implies that $Q$ should scale with $O(\sqrt{N})$ to ensure a maximum blocking probability of $1 / \nu$. Note that if $\sqrt{2 N \ln \nu}+\frac{1-\alpha^{N+1}}{1-\alpha}>N$, then the blocking probability of $1 / \nu$ is not achievable. A related question of interest is how $Z_{N}$ scales as $N \rightarrow \infty$.

Proposition 2 (Connection Requests): For an $\alpha$-prioritized cluster with arbitrarily large $N$, there exists an $N_{0}$ dependent 
on $\alpha$ such that for all $N>N_{0}$, the number of nodes that express a desire to connect to the master node in a cluster can be bounded above w.h.p. by

$$
Z_{N} \leq \min \left\{N, N \sqrt{2 \ln (1 / \alpha)}+\frac{1}{1-\alpha}\right\}
$$

For example, let $\alpha=0.95$ for a cluster. Then there exists an $N_{o}$ such that if the cluster has $N>N_{o}$ nodes, then the number of nodes that try to connect to the master node is bounded above by $0.323 N+20$ w.h.p. The two propositions above are established in the sequel.

Denote by $B_{i}$ the event that node $i$ tries to connect to the master node. Without loss of generality, we assume that $\left\{B_{i}\right\}_{i=1}^{N}$ are identically and independently distributed (i.i.d.). Let $\mathcal{B}_{N}$ be the sigma algebra formed by the events $\left\{B_{1}, B_{2}, \ldots, B_{N}\right\}$. Then we have $Z_{N}=\sum_{i=1}^{N} I\left(B_{i}\right)$, where $I(\cdot)$ is the indicator function. If there are $k$ terminal nodes in the cluster, then $Z_{k}=\sum_{i=1}^{k} I\left(B_{i}\right)$. Recall the following lemma.

Lemma 1 (Azuma's Inequality [14]): Let $\left\{Y_{0}, Y_{1}, Y_{2}, \ldots\right\}$ be a martingale sequence such that for each $k,\left|Y_{k}-Y_{k-1}\right| \leq$ $c_{k}$, where $c_{k}$ depends on $k$. Then, for all $k \geq 1$ and for any $\mu>0$,

$$
\operatorname{Pr}\left(Y_{k} \geq \mu\right) \leq \exp \left(-\frac{\mu^{2}}{2 \sum_{j=1}^{k} c_{j}^{2}}\right)
$$

Motivated by the discussion in [15], we introduce a martingale sequence $Y_{k}$ and utilize the above lemma to obtain a bound on $Z_{N}$. Let $Y_{k}=Z_{k}-\sum_{i=1}^{k} \operatorname{Pr}\left(B_{i}\right)$ with $Y_{0}=1$. It can be shown that $Y_{k}$ is a martingale because

$$
\begin{aligned}
\mathbb{E}\left[Y_{k+1} \mid Y_{k}\right] & =\mathbb{E}\left[Y_{k+1} \mid \mathcal{B}_{k}\right] \\
& =\mathbb{E}\left(\left(\sum_{i=1}^{k+1} I\left(B_{i}\right)-\sum_{i=1}^{k+1} \operatorname{Pr}\left(B_{i}\right)\right) \mid \mathcal{B}_{k}\right) \\
& \left.=Y_{k}+\mathbb{E}\left[I\left(B_{k+1} \mid B_{k}\right)\right]-\mathbb{E}\left\{\mathbb{E}\left[I\left(B_{k+1}\right)\right] \mid \mathcal{B}_{k}\right]\right\} \\
& =Y_{k}
\end{aligned}
$$

Moreover, it can be shown that $\left|Y_{k}-Y_{k-1}\right| \leq 1$. Now applying Azuma's inequality with $\mu=\sqrt{2 k \ln \nu}$, we get

$$
\operatorname{Pr}\left(Y_{k} \geq \sqrt{2 k \ln \nu}\right) \leq \frac{1}{\nu}, \quad k \geq 1
$$

or equivalently,

$$
\operatorname{Pr}\left(\sum_{i=1}^{k} I\left(B_{i}\right)-\sum_{i=1}^{k} \operatorname{Pr}\left(B_{i}\right) \geq \sqrt{2 k \ln \nu}\right) \leq \frac{1}{\nu}, k \geq 1
$$

Noting that the cluster is an $\alpha$-prioritized cluster, and using the fact that $\left\{B_{i}\right\}$ are independent of each other, we obtain

$$
\sum_{i=1}^{k} \operatorname{Pr}\left(B_{i}\right) \leq \frac{1-\alpha^{k+1}}{1-\alpha}
$$

Substituting (8) into (7), we have

$$
\operatorname{Pr}\left(Z_{k} \geq \sqrt{2 k \ln \nu}+\frac{1-\alpha^{k+1}}{1-\alpha}\right) \leq \frac{1}{\nu}
$$

Since there are $N$ terminal nodes in a cluster, Proposition 1 is established.
Consider again Azuma's inequality but choose $\mu=$ $k \sqrt{2 \ln (1 / \alpha)}$, then

$$
\operatorname{Pr}\left(Y_{k} \geq k \sqrt{2 \ln (1 / \alpha)}\right) \leq \alpha^{k}, \quad k \geq 1
$$

Summing the above inequality over $k$, we get

$$
\sum_{k=0}^{\infty} \operatorname{Pr}\left(Y_{k} \geq k \sqrt{2 \ln (1 / \alpha)}\right) \leq \frac{1}{1-\alpha}<\infty
$$

From the Borel Cantelli Lemma [15], we conclude that the event $\left\{Y_{k} \geq k \sqrt{2 \ln (1 / \alpha)}\right\}$ cannot occur infinitely often. Thus, for sufficiently large $k$, say $k \geq N_{o}$, we have $Y_{k} \leq$ $k \sqrt{2 \ln (1 / \alpha)}$ w.h.p. Hence, when the number of terminals in a cluster is $N$, we get

$$
Z_{N} \leq N \sqrt{2 \ln (1 / \alpha)}+\frac{1}{1-\alpha} \text { w.h.p. }
$$

which leads to Proposition 2.

\section{B. Distance-Based Probabilistic Connection}

We now consider a probabilistic connection scheme that is based on the distance between nodes. Assuming the master node knows the locations of all terminal nodes, the probability that node $i$ successfully connects to the master node can be modelled in terms of its distance from the master node as

$$
\operatorname{Pr}\left(B_{i}\right)=F\left(d_{i}\right)=\left(\frac{d_{0}}{d_{i}}\right)^{1 / \delta}, d_{0} \leq d_{i} \leq d_{M}
$$

where $d_{0}$ and $d_{M}$ are respectively the minimum and maximum distances ${ }^{3}$ between the transmitting node and the master node, and $\delta$ is a user defined parameter $(0.5<\delta<1)$. $d_{i}$ is the distance between the terminal node and its intended master node.

Denote the position of the master node at time $t$ by $X(t)$. Considering the energy requirement for many important tasks, it is reasonable to assume that $\{X(t)\}$ is stationary and ergodic with uniform distribution on the disc. Since the nodes are i.i.d. with a uniform distribution, conditional on $X(t)=x$, the cumulative probability density function of $d_{i}$ is given by

$$
\operatorname{Pr}\left(d_{i} \leq r \mid X(t)=x\right)=c(x) \frac{r^{2}-d_{0}^{2}}{d_{M}^{2}-d_{0}^{2}}, \quad d_{0} \leq r \leq d_{M}
$$

where $c(x)(0<c(x) \leq 1)$ is a constant factor dependent on $x$ and is used to account for edge effects. If the master node is near the periphery of the cluster, then $c(x)<1$; otherwise, $c(x)=1$. Differentiating the above equation, the probability distribution function (p.d.f.) of $d_{i}$ follows

$$
f_{d_{i}}(r \mid X(t)=x)=\frac{2 r c(x)}{d_{M}^{2}-d_{0}^{2}}, \quad d_{0} \leq r \leq d_{M}
$$

Proposition 3 (Number of Time Slots): In the distancebased probabilistic connection scenario defined by (13), $Q$ should scale with $O(N)$ in order to ensure a constant blocking probability ${ }^{4}$.

${ }^{3}$ For the requirement of network connectivity [16], we usually have $d_{0} \geq$ $\kappa \sqrt{\frac{A \log N}{N}}$, which ensures that no node in the cluster is isolated w.h.p. Note that $\kappa$ is a certain constant.

${ }^{4}$ The notation $y=O(g(N))$ denotes that there exits a constant $\kappa$ such that $\lim _{N \rightarrow \infty} \frac{y}{g(N)} \leq \kappa$. 
Proof: When $Z_{N}>Q$, the master node chooses $Q$ closest nodes for transmission. Since $Z_{N}$ takes nonnegative values, the blocking probability can be bounded above as

$$
\operatorname{Pr}\left(Z_{N}>Q\right) \leq \frac{\mathbb{E}\left(Z_{N}\right)}{Q}=\frac{\mathbb{E}\left(\sum_{i=1}^{N} I\left(B_{i}\right)\right)}{Q}
$$

where (15) follows from the Markov inequality [17][18]. Using the fact that $\left\{B_{i}\right\}_{i=1}^{N}$ are independently and identically distributed, we have

$$
\begin{aligned}
\mathbb{E}\left(\sum_{i=1}^{N} I\left(B_{i}\right)\right) & =N \mathbb{E}\left(I\left(B_{1}\right)\right) \\
& =N \int_{d_{0}}^{d_{M}} F(r) f_{d_{i}}(r \mid X(t)=x) d r \\
& =O(N)
\end{aligned}
$$

Combining (15) and (16) yields Proposition 3.

In this network scenario, in order to ensure a constant blocking probability $1 / \nu, Q$ should scale with $O(N)$. This is because the connection probability of a terminal node is completely determined by its distance from the intended master node.

\section{Throughput CAPACITY}

Without loss of generality, the per node throughput is defined as the time average of the number of bits per second that can be transmitted from every source node to its destination. Specifically, we adopt the following asymptotic notion as defined in [1] and [19].

Definition 4 (Feasible Per Node Throughput): Let $M_{i}(t)$ denote the amount of information originated from node $i$ to its destinations at time $t$. Given the random locations of the source and destination nodes, we shall say a long-term per node throughput of $\lambda(N)$ bits per second is feasible if there is a spatial and temporal scheduling policy $\pi$ such that

$$
\liminf _{T \rightarrow \infty} \frac{1}{T} \sum_{t=1}^{T} M_{i}(t) \geq \lambda(N)
$$

For each transmission, we assume that the transceiver is able to adaptively control the transmitting power level so that the SINR can maintain a value of at least $\gamma$. In the capacity analysis below, we limit our attention to intra-cluster communications.

\section{A. Single-Hop REACA}

In the single-hop REACA network, terminal nodes send data packets to their master node. Since each cluster can obtain a common transmission rate of $R$ and there are $M$ simultaneous transmissions at any moment, the aggregate transmission rate is $M R$. This rate is shared by the $N_{t}$ nodes in the network, and thus, the per node throughput capacity is given as $\Theta\left(M R / N_{t}\right)$.

\section{B. Multi-Hop REACA}

In the following, we analyze the multi-hop REACA network.
1) Packet Routing: We first investigate the routing behavior of packets within a cluster. Fig. 3 shows an example of routing a packet from a source node to the destination. The packets travel almost in the same direction toward the destination at each step. To derive the number of hops for each packet, we partition the cluster into many cells with an equal area $D$, as shown in Fig. 3. Before proceeding, we need the following lemma.

Lemma 2 (Cluster Partitioning): Consider a cluster that is partitioned into many cells with an equal area $D$. There exits a deterministic positive constant $\mu$, such that if $D \geq \mu \log N / N$, then every cell contains at least one node w.h.p.

Proof: Consider $N+1$ nodes i.i.d. in a cluster $\mathcal{A}$ of area $A$. Any cell $\mathcal{D}$ is a subset of $\mathcal{A}$, i.e., $\mathcal{D} \subset \mathcal{A}$. Recall the coverage result given in [20]. When $N$ is large enough, the number of nodes in a given area $D$ is Poisson distributed with mean $\rho D$, where $\rho=(N+1) / A$ is the node density. That is, the probability that a cell $\mathcal{D}$ contains $k$ nodes is

$$
\operatorname{Pr}(\text { There are } k \text { nodes located in } \mathcal{D})=\frac{(\rho D)^{k}}{k !} e^{-\rho D}
$$

Given a cell $\mathcal{D}$, the probability that it contains at least one node is given by

$$
\operatorname{Pr}(k \geq 1 \mid \mathcal{D})=1-\operatorname{Pr}(k=0 \mid \mathcal{D})=1-e^{-\rho D}
$$

Let $D=\mu \log N / N$, then

$$
\operatorname{Pr}(k \geq 1 \mid \mathcal{D}) \geq 1-e^{-\mu \log N / A} \longrightarrow 1 \text { as } N \longrightarrow \infty
$$

This completes the proof.

Corollary 1 (Cluster Coverage): As the density of nodes $\rho=(N+1) / A$ increases, the cluster is fully covered w.h.p.

The above corollary says that any non-zero area around the final destination node can be reached by a packet w.h.p. when the density of nodes is large enough.

Consider each of the $N$ terminal nodes in a cluster generating $M_{i}(t)$ bits to their destinations at time $t$. For these $N$ S-D pairs, the following theorem estimates how many routes will pass through a certain node in the cluster.

Theorem 1 (Number of Routes Through a Node): At any time, in a cluster with $N$ terminal nodes, the number of generated routes that will pass through a certain node is $\Theta(\sqrt{N / \log N})$.

Proof: We first partition the cluster into many cells with a common area $D \geq \mu \log N / N$ so that each cell contains at least one node w.h.p. Consider an S-D pair $i$ at any time $t$. The number of hops needed for the packet to move from the source to the destination is denoted by $h_{i}$. The distance between the source and destination nodes of S-D pair $i$ is $l_{i}$. Since the routes are nearly straight lines, the number of hops for S-D pair $i$ can be written as $h_{i}=\Theta\left(l_{i} / \sqrt{D}\right)$. For any cell $j$, we define a Bernoulli random variable $Y_{i j}$ as

$Y_{i j}= \begin{cases}1 & \text { if the route of } \mathrm{S}-\mathrm{D} \text { pair } i \text { intersects cell } j \\ 0 & \text { otherwise }\end{cases}$

where $\operatorname{Pr}\left(Y_{i j}=1\right)=1-\operatorname{Pr}\left(Y_{i j}=0\right)=p_{i j}$. Then $Y_{j}=$ $\sum_{i=1}^{N} Y_{i j}$ is the total number of routes that intersect cell $j$. Its 


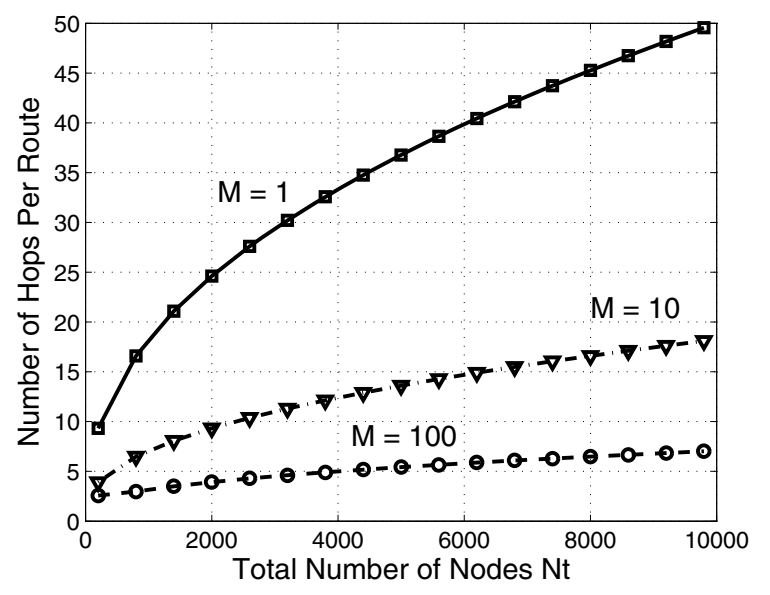

Fig. 4. The number of hops per route v.s. the total number of nodes in the network. $M$ is the number of clusters in the whole network.

expected value is

$$
\mathbb{E}\left(Y_{j}\right)=\mathbb{E}\left(\sum_{i=1}^{N} Y_{i j}\right)=N \mathbb{E}\left(Y_{i j}\right)=\frac{N \mathbb{E}\left(h_{i}\right)}{A / D}
$$

(18) follows the assumption that the source and destination of S-D pair $i$ are independently and uniformly distributed in the cluster. Because $d_{0} \leq l_{i} \leq d_{M}$, we have

$$
\mathbb{E}\left(h_{i}\right)=\mathbb{E}\left(l_{i}\right) \Theta\left(\frac{1}{\sqrt{D}}\right)=\Theta\left(\frac{1}{\sqrt{D}}\right)
$$

Substituting (19) into (18), we have

$$
\mathbb{E}\left(Y_{j}\right)=\Theta(N \sqrt{D})=\Theta(\sqrt{N \log N})
$$

As implied by the Chernoff bound [21] for independently distributed Bernoulli random variables,

$$
\operatorname{Pr}\left\{Y_{j}>(1+\epsilon) \mathbb{E}\left(Y_{j}\right)\right\}<e^{-\epsilon^{2} \mathbb{E}\left(Y_{j}\right) / 4}, \text { for } \epsilon<2 e-1
$$

and

$$
\operatorname{Pr}\left\{Y_{j}>(1+\epsilon) \mathbb{E}\left(Y_{j}\right)\right\}<2^{-\epsilon \mathbb{E}\left(Y_{j}\right)}, \text { for } \epsilon>2 e-1
$$

Let $\epsilon=2 \sqrt{\log N / \mathbb{E}\left(Y_{j}\right)}$, we have

$$
\operatorname{Pr}\left\{Y_{j}>(1+\epsilon) \mathbb{E}\left(Y_{j}\right)\right)<\max \left(\frac{1}{N}, 2^{-2 \sqrt{\mathbb{E}\left(Y_{j}\right) \log N}}\right)(21)
$$

where the right hand side goes to zero as $N \longrightarrow+\infty$. On the other hand, we have

$$
\operatorname{Pr}\left\{Y_{j}<(1-\epsilon) \mathbb{E}\left(Y_{j}\right)\right\}<e^{-\epsilon^{2} \mathbb{E}\left(Y_{j}\right) / 2}=\frac{1}{N^{2}}
$$

which also goes to zero as $N \longrightarrow+\infty$.

Combining (21) and (22) yields

$$
Y_{j}=\mathbb{E}\left(Y_{j}\right)+o\left(\mathbb{E}\left(Y_{j}\right)\right) \text { w.h.p. }=\Theta(\sqrt{N \log N}) \text { w.h.p. }
$$

where $f(N)=o(g(N))$ means that $\lim _{N \rightarrow \infty} f(N) / g(N)=$ 0 .

Since the number of cells in a cluster is given as $\Theta(A / D)=$ $\Theta(N / \log N)$, the number of nodes per cell is $\Theta(\log N)$. Considering that the source and destination nodes are i.i.d., from a long term average point of view, the routes through a cell are evenly distributed on each node of this cell. Therefore, the number of routes that pass through each node is given by

$$
\frac{Y_{j}}{\Theta(\log N)}=\Theta\left(\sqrt{\frac{N}{\log N}}\right) \text { w.h.p. }
$$

This completes the proof.

The following corollary bounds the expected number of hops for each packet.

Corollary 2 (Number of Hops Per Route): The mean number of hops that a packet takes from its source to the destination is $\Theta(\sqrt{N / \log N})$.

Proof: From (19), we have

$$
\mathbb{E}\left(h_{i}\right)=\Theta\left(\frac{1}{\sqrt{D}}\right)=\Theta\left(\sqrt{\frac{N}{\log N}}\right)
$$

We plot the function (23) in Fig. 4. It shows that in a non-clustered network $(M=1)$, the number of hops per route increases rapidly as the number of nodes in the network increases. When the network is partitioned into many nonoverlapping clusters $(M=10$ or $M=100)$, the number of hops increases slowly with respect to the total number of nodes in the network because the routing is restricted within the clusters.

2) The Distance Between the Source and Destination Nodes: The distance that a packet travels from its source to the destination node within the cluster is a function of the number of hops in routing, and can be determined asymptotically by the number of nodes in the cluster as shown in Lemma 3 below.

Lemma 3 (Mean Travel Distance): The mean distance $\bar{L}$ that a packet travels within a cluster from its source node to the destination is $O\left(\sqrt[4]{\frac{1}{N \log N}}\right)$.

Proof: Consider a large time $T$ seconds, during which the cluster transports a total number of $\lambda N T$ bits. For bit $b$ $(1 \leq b \leq \lambda N T)$, we denote by $h(b)$ the number of hops it takes to reach the destination, and let $r(b, h)$ represent the length of hop $h, 1 \leq h \leq h(b)$. Then, it follows that

$$
\sum_{b=1}^{\lambda N T} \sum_{h=1}^{h(b)} r(b, h) \geq \lambda N \bar{L} T
$$

Because $d_{0} \leq r(b, h) \leq d_{M}$, and summing over the $R T$ bits transmitted during the $T$ seconds, we also have

$$
\sum_{b=1}^{\lambda N T} \sum_{h=1}^{h(b)} r^{2}(b, h) \leq R T d_{M}^{2}
$$

The total number of hops taken by the $\lambda N T$ bits is denoted by $H=\sum_{b=1}^{\lambda N T} h(b)$. By the convexity of quadratic functions, we obtain

$$
\left(\sum_{b=1}^{\lambda N T} \sum_{h=1}^{h(b)} \frac{r(b, h)}{H}\right)^{2} \leq \sum_{b=1}^{\lambda N T} \sum_{h=1}^{h(b)} \frac{r^{2}(b, h)}{H}
$$

Combining (24), (25), and (26) together gives

$$
\lambda N \bar{L} \leq \sqrt{\frac{H R}{T}} d_{M} .
$$




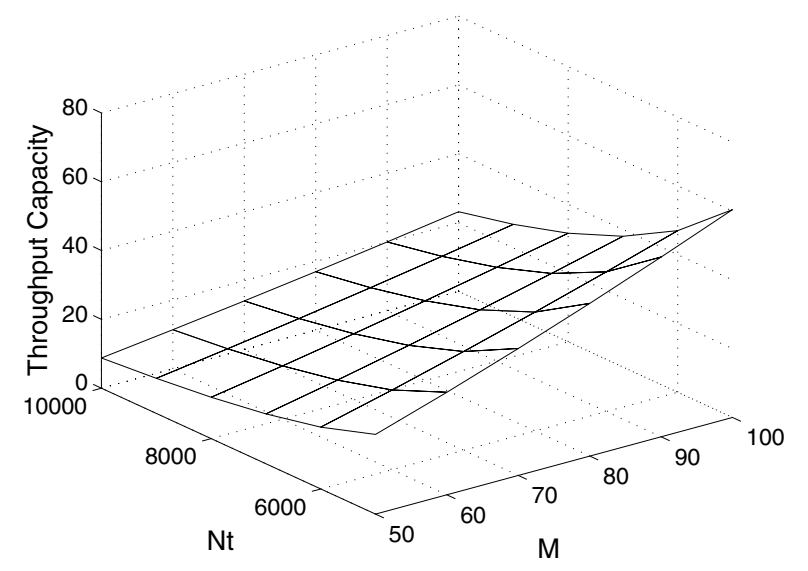

Fig. 5. Throughput capacity in wireless clustered networks.

Denote the variance of $h(b)$ by $\sigma_{h}^{2}$. Assuming that the hops are i.i.d. and $\sigma_{h}^{2}<+\infty$, by the weak law of large numbers [17] (pp. 69-71), we get for any $\varepsilon>0$,

$\operatorname{Pr}\left(\left|\frac{H}{\lambda N T}-\mathbb{E}(h(b))\right|>\varepsilon\right) \leq \frac{1}{\varepsilon^{2}} \frac{\sigma_{h}^{2}}{\lambda N T} \longrightarrow 0$ as $T \longrightarrow \infty$

Thus, when $T$ is large enough,

$$
\mathbb{E}(h(b))=\frac{H}{\lambda N T} \quad \text { w.h.p. }
$$

Substituting (28) into (27), we get

$$
\bar{L} \leq d_{M} \sqrt{\frac{R \mathbb{E}(h(b))}{\lambda N}} \text { w.h.p. }
$$

Because the number of hops $h$ that a packet takes is the same order as $h(b)$, i.e., $h=h(b)$, it holds that $\bar{L}=O(\sqrt{\mathbb{E}(h) / N})$ w.h.p. Combined with Corollary 2, the result is established.

Rearranging (29) gives

$$
\mathbb{E}(h) \geq \frac{\lambda N \bar{L}^{2}}{R d_{M}^{2}} \quad \text { w.h.p. }
$$

As implied by (30), when the number of nodes, transmission rate, and per node throughput are specified, the mean number of hops per route is lower bounded by a function of the square of the distance that a packet travels. With this information, one can predict how the packets will be routed within the cluster given the distance between two nodes.

3) Throughput Capacity: Consider that each cluster transmits at a rate of $R$. This rate should be shared by $N$ nodes, each of which generates traffic at a rate of $\lambda(N)$, i.e.,

$$
N \lambda(N) \mathbb{E}(h)=R .
$$

Substituting (23) into (31), we obtain that the per node throughput capacity

$$
\lambda(N)=\Theta\left(R \sqrt{\frac{\log N}{N^{3}}}\right)
$$

is feasible w.h.p. Considering the $N_{t} \approx M N$ nodes in the entire network, we obtain the following asymptotic per node throughput capacity.
Theorem 2 (Per Node Throughput Capacity): For a largescale multi-hop REACA network with $N_{t}$ nodes organized into $M$ clusters, the per node throughput capacity is given by

$$
\Theta\left(R \sqrt{\frac{M^{3}\left(\log N_{t}-\log M\right)}{N_{t}^{3}}}\right) \quad \text { w.h.p. }
$$

It can be shown that given the number of nodes, the per node throughput capacity of the network increases with the number of clusters, as illustrated by a numerical example in Fig. 5 . The improvement is mainly due to the fact that clustering greatly limits the number of hops in routing and thus reduces the relaying burden carried by each node. Another important observation is that the short-range communication imposed by the clustered structure decreases the interference and allows for more simultaneous transmissions in the entire network.

One may want to consider the effect of traffic sent by the master node to the BS. For simplicity, the communication between the clusters and BS is scheduled by a TDMA scheme and has a transmission rate of $R_{1}$. The throughput capacity between a cluster (or master node) and the BS is $\Theta\left(\frac{R_{1}}{M T_{m}}\right)$, which vanishes when $T_{m}$ is large. Therefore, we can assume that this amount of traffic is negligible in the asymptotic analysis.

Compared with the non-clustered wireless network considered in [1], which has a per node throughput capacity of $\Theta\left(R / \sqrt{N_{t} \log N_{t}}\right)$, the single-hop REACA network will obtain a better throughput capacity if the number of clusters is large enough, i.e., $M>\sqrt{\frac{N_{t}}{\log N_{t}}}$. For the multi-hop REACA network, it is sufficient that $\sqrt[3]{\frac{N_{t}^{2}}{\log N_{t}}}<M<\frac{N_{t}}{2}$ makes it outperform the non-clustered wireless network. Therefore, clustered networks can achieve throughput improvement by taking advantage of traffic locality and in-network data aggregation. If the degree of traffic locality or data aggregation is low, the non-clustered wireless network might outperform the clustered networks.

\section{POWER CONSUMPTION}

In this section, we study the power consumption of the REACA network and compare its performance with that of non-clustered networks.

\section{A. Multi-Hop vs. Single-Hop}

In wireless networks, it is in general preferable to use multiple short-range transmissions rather than a long-range transmission. Consider that a packet can be transmitted from the source to the destination through $m$ hops, which have distances $L_{1}, L_{2}, \cdots, L_{m}$. Denote the distance between the source and destination nodes by $L$. If the route is nearly a straight line, then $L \approx \sum_{i=1}^{m} L_{i}$. Provided that the transceiver works on a fixed transmission rate $R$ with a frequency band of bandwidth $W$, the required signal-to-noise ratio (SNR) of each hop is given by

$$
\gamma=2^{R / W}-1=\frac{P_{i} G_{i}}{\sigma_{N}^{2}}, \quad 1 \leq i \leq m
$$




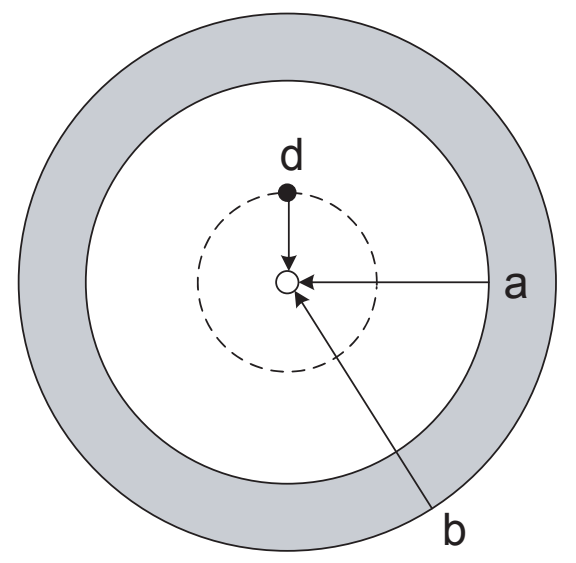

Fig. 6. Annulus between distances $a$ and $b$ from the master node.

where $\sigma_{N}^{2}$ is the Gaussian noise level, $P$ is the transmission power, and $G$ is the channel gain given in (2). Substituting (2) into the above equation, we get

$$
L_{i}=\left(\frac{S_{0} 10^{\eta / 10}}{\gamma \sigma_{N}^{2}} P_{i}\right)^{1 / \beta}
$$

Since $L \approx \sum_{i=1}^{m} L_{i}$, assuming the same noise level $\sigma_{N}^{2}$ at each receiver and the same shadowing factor over each channel, we have

$$
P^{1 / \beta}=\sum_{i=1}^{m} P_{i}^{1 / \beta}
$$

Since $\beta>2$, and due to convexity, it follows that

$$
P=\left(\sum_{i=1}^{m} P_{i}^{1 / \beta}\right)^{\beta} \geq \sum_{i=1}^{m} P_{i}
$$

Therefore, the power needed for transmission over a single hop of length $L$ is greater than the sum of power over $m$ short hops $L_{i}, 1 \leq i \leq m$. Thus, multi-hop routing results in significant power saving compared with the single direct transmission. We should emphasize that the power efficiency is achieved at the cost of increasing the routing delay, which scales with $O(m)$.

\section{B. REACA Networks}

For REACA networks, we will show that there exists $P_{0}>0$ such that if all the transmitting nodes transmit at an equal power level $P>P_{0}$, then each cluster can obtain a common transmission rate of $R$. This can be accomplished by a simple distributed power control scheme in which the receivers measure the interference and tell their transmitters how to adjust the power levels. This process is iterated until all the transceivers reach equilibrium. More sophisticated power control algorithms can be found in [22] and the references therein.

In a cluster, denote the distance between a node and its master node by $d\left(d_{0} \leq d \leq d_{M}\right)$. Within the distance of $(1+\varepsilon) d$ from the master, where $\varepsilon>0$ is a guard parameter for safety margin, we assume that the number of potential interfering nodes is upper bounded by some constant $n_{1}$. We may enforce a spatial and temporal scheduling scheme to ensure none of interfering nodes from neighboring clusters transmits simultaneously within the distance of $(1+\varepsilon) d$ from the master node. In this way, all the remaining interfering nodes would be from outside the distance of $(1+\varepsilon) d$ from the intended master of the node.

Consider the annulus of all points lying within a distance between $a$ and $b$ from the master node, as shown in Fig. 6 . The area of the annulus is given by

$$
\pi\left(b^{2}-a^{2}\right)=\pi(2 k+1)(1+\varepsilon)^{2} d^{2}
$$

where $a=k(1+\varepsilon) d$ and $b=(k+1)(1+\varepsilon) d$ for $k=1,2, \cdots$, and this area contains no more than $\pi(2 k+1)(1+\varepsilon)^{2} d^{2} / A$ clusters, each of which has an interfering transmitting node. Thus, by summing over all interfering nodes, i.e., over $k$, the interference at the master node can be bounded above as

$$
\begin{aligned}
I_{i}(t) & =\sum_{j \neq i} P_{j}(t) G_{j i}(t) \\
& \leq \frac{\pi c^{\prime} P}{A(1+\varepsilon)^{\beta-2} d^{\beta-2}}\left(\sum_{k=1}^{\infty} \frac{2 k+1}{k^{\beta}}\right) \\
& \leq \frac{\pi c^{\prime} P}{A(1+\varepsilon)^{\beta-2} d^{\beta-2}}\left(3+\frac{2}{\beta-2}+\frac{1}{\beta-1}\right)(36)
\end{aligned}
$$

where $c^{\prime}=S_{0} 10^{\eta / 10}$.

Therefore, for large enough power, the SINR at the master node is lower bounded by

$$
\begin{aligned}
\gamma & \triangleq \lim _{P \rightarrow \infty} \frac{\frac{c^{\prime} P}{d^{\beta}}}{\frac{\pi c^{\prime} P}{A(1+\varepsilon)^{\beta-2} d^{\beta-2}}\left(3+\frac{2}{\beta-2}+\frac{1}{\beta-1}\right)+\sigma_{i}^{2}} \\
& =\frac{A(1+\varepsilon)^{\beta-2}}{\pi d^{2}\left(3+\frac{2}{\beta-2}+\frac{1}{\beta-1}\right)}
\end{aligned}
$$

This result indicates that when the guard parameter $\varepsilon$ is specified, there is a large enough power level $P$ to transmit packets such that the SINR can attain a desired level $\gamma$. Subsequently, the transmissions in the cluster would be successfully received by the master node.

We now investigate how the per node power consumption $p$ will scale with $N_{t}$.

Lemma 4 (Power Consumption for REACA Networks): In the REACA network described above, the average per node power consumption can be estimated as

$$
\mathbb{E}(p)=O\left(\frac{M}{N_{t}} \gamma\right)
$$

Proof: From (1), we have

$$
P_{i}(t)=\frac{\gamma}{G_{i i}(t)}\left(\sum_{j \neq i} P_{j}(t) G_{j i}(t)+\sigma_{i}^{2}\right)
$$

Since the channel gain $G_{i i}(t)$ depends only on the transmission distance and the physical environment, taking expectations of both sides of (39) gives

$$
\begin{aligned}
\mathbb{E}\left(P_{i}\right) & =\mathbb{E}\left(\frac{\gamma}{G_{i i}(t)}\right) \mathbb{E}\left(\sum_{j \neq i} P_{j}(t) G_{j i}(t)+\sigma_{i}^{2}\right) \\
& \leq \mathbb{E}\left(\frac{\gamma}{G_{i i}(t)}\right)\left(\frac{c^{\prime}}{\gamma d^{\beta}} \mathbb{E}\left(P_{i}\right)+\sigma_{i}^{2}\right) \\
& \leq \frac{c^{\prime} c^{\prime \prime}}{d^{\beta}} \mathbb{E}\left(P_{i}\right)+\gamma c^{\prime \prime} \sigma_{i}^{2}
\end{aligned}
$$




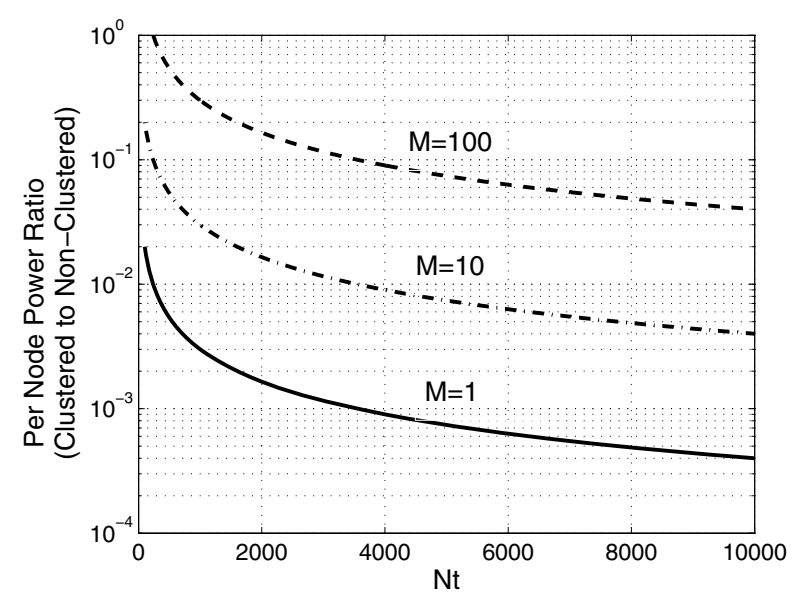

Fig. 7. The per node power consumption ratio of the proposed network to the non-clustered network.

where (40) follows from

$$
\begin{aligned}
\mathbb{E}\left(\frac{\gamma}{G_{i i}(t)}\right) & =\int_{d_{0}}^{d_{M}} \mathbb{E}\left[\frac{\gamma}{G_{i i}(t)} \mid d_{i}\right] f_{d_{i}}(r \mid X(t)=x) d r \\
& \leq \frac{\gamma S_{0}\left(d_{M}^{\beta+2}-d_{0}^{\beta+2}\right)}{\left(d_{M}^{2}-d_{0}^{2}\right)(\beta+2)} e^{\left(\sigma_{\eta} \ln 10\right)^{2} / 200} \\
& =\gamma c^{\prime \prime}
\end{aligned}
$$

Rearranging (40), we obtain

$$
\mathbb{E}\left(P_{i}\right) \leq \frac{\gamma c^{\prime \prime} \sigma_{i}^{2} d^{\beta}}{d^{\beta}-c^{\prime} c^{\prime \prime}} \leq \frac{\gamma c^{\prime \prime} \sigma_{i}^{2} d_{0}^{\beta}}{d_{0}^{\beta}-c^{\prime} c^{\prime \prime}}
$$

Then, it can be easily seen that $\mathbb{E}\left(P_{i}\right)=O(\gamma)$. As a consequence, the average per node power consumption can be given by

$$
\mathbb{E}(p)=\frac{\mathbb{E}\left(P_{i}\right)}{N+1}=O\left(\frac{M}{N_{t}} \gamma\right)
$$

Lemma 4 shows that the power consumed by the network depends on the number of clusters as well as on the simultaneous transmissions. Furthermore, if the number of clusters $M$ is a constant, the per node power consumption is of the order of $1 / N_{t}$. With high degree of traffic locality and in-network data aggregation (i.e., large $T_{m}$ ), the power used to transmit information from the master node to the $\mathrm{BS}$ is negligible in Lemma 4.

\section{Non-Clustered Wireless Networks}

We now investigate the per node power consumption in a non-clustered multi-hop routing network without clustering as described in [1].

Lemma 5 (Power Consumption for Non-clustered Networks) In the non-clustered wireless network, the expected per node power consumption can be estimated as $\mathbb{E}(p)=O\left(\gamma / \log N_{t}\right)$.

Proof: Consider the protocol model in [1], in which all source nodes transmit with a common distance $r\left(N_{t}\right)$. Due to the requirement of network connectivity [16], the common transmission distance should satisfy

$$
r\left(N_{t}\right) \geq \sqrt{\frac{\log N_{t}}{\pi N_{t}}}
$$

which ensures that no node in the network will be isolated w.h.p. To successfully transmit packets from node $X_{i}$ to another node $X_{j}$ at a distance $\left|X_{i}-X_{j}\right|$, where $\left|X_{i}-X_{j}\right| \leq$ $r\left(N_{t}\right)$, there should be no node simultaneously transmitting within the distance $(1+\varepsilon) r\left(N_{t}\right)$ from $X_{j}$. In this way, each transmission consumes an area of at least $\pi r^{2}\left(N_{t}\right)$ because $\varepsilon>0$. Therefore, provided that the entire network is deployed in a disc of unit area in $\mathcal{R}^{2}$, the total number of simultaneous transmissions can be bounded above by

$$
\frac{1}{\pi r^{2}\left(N_{t}\right)} \leq \frac{N_{t}}{\log N_{t}}
$$

Then the per node power consumption can be given by

$$
\mathbb{E}(p) \leq \frac{1}{N_{t}} \frac{N_{t}}{\log N_{t}} \mathbb{E}(P)=\frac{\mathbb{E}(P)}{\log N_{t}}
$$

where $\mathbb{E}(P)$ is the average power consumption for each transmission. Using similar techniques to Lemma 4, it can be shown that $\mathbb{E}(P)=O(\gamma)$ and the result follows.

For the physical model in [1], in which each node transmits at a common power level, it can be shown that if $\varepsilon$ is properly chosen then the above result holds for a large enough common power level $P$.

By comparing the above results, we find that the clusterbased structure is more power efficient than the non-clustered network provided $M \leq \frac{N_{t}}{\log N_{t}}$. Given a large set of wireless network nodes, each node of the non-cluster network requires more power, by a factor of at least $O\left(\frac{N_{t}}{M \log N_{t}}\right)$, than that of the clustered structure. The reason is that the cluster-based network limits the number of simultaneous transmissions nearby by clustering, and thus reduces energy dissipation. Besides, due to spatial separation, the transceivers of the clustered network can achieve the same SINR with less power compared with the non-clustered model. Fig. 7 illustrates the per node power consumption ratio of the clustered network to the non-clustered network.

Taking into consideration the throughput capacity and power consumption together, we find that the preferable range for the number of clusters in single-hop REACA networks is $\sqrt{\frac{N_{t}}{\log N_{t}}}<M<\frac{N_{t}}{\log N_{t}}$. In multi-hop REACA networks, the network is expected to utilize bandwidth and energy efficiently if $\sqrt[3]{\frac{N_{t}^{2}}{\log N_{t}}}<M<\frac{N_{t}}{\log N_{t}}$.

\section{CONCLUSION}

In this paper, we have developed REACA, a robust energyaware clustering protocol architecture for large-scale wireless sensor networks. We examined its performance in terms of quality-of-service, throughput capacity, and power consumption. It has been shown that clustering can utilize bandwidth and energy efficiently by taking advantage of spatial separation and traffic locality. In particular, we focus on intracluster communications without considering communications between clusters and the BS. Nevertheless, in some scenarios, one may have to consider inter-cluster traffic. An interesting extension is to optimally determine the link capacity between the master nodes and BS according to a specific aggregation function so that the data traffic can fully exploit the available bandwidth and network architecture. Overall, the results 
obtained in this paper provide useful insights for design and implementation of large-scale WSNs.

\section{REFERENCES}

[1] P. Gupta and P. R. Kumar, "The capacity of wireless networks," IEEE Trans. Inform. Theory, vol. 46, no. 2, pp. 388-404, Mar. 2000.

[2] I. F. Akyildiz, W. Su, Y. Sankarasubramaniam, and E. Cayirci, "Wireless sensor networks: A survey," Computer Networks, vol. 38, pp. 393-422, Mar. 2002.

[3] J. N. Al-Karaki and A. E. Kamal, "Routing techniques in wireless sensor networks: A survey," IEEE Wireless Commun., vol. 11, no. 6, pp. 6-28, Dec. 2004.

[4] C. Lin and M. Gerla, "Adaptive clustering for mobile wireless networks," IEEE J. Select. Areas Commun., vol. 15, pp. 1265-1275, Sep. 1997.

[5] D. Baker, A. Ephremides, and J. Flynn, "The design and simulation of a mobile radio network with distributed control," IEEE J. Select. Areas Commun., vol. 2, pp. 226-237, Jan. 1984.

[6] V. Kawadia and P. R. Kumar, "Power control and clustering in ad hoc networks," in Proc. IEEE INFOCOM, Mar. 2003, vol. 1, pp. 459-469.

[7] A. Subramanian and A. H. Sayed, "Joint rate and power control algorithms for wireless networks," IEEE Trans. Signal Processing, vol. 53, no. 11, pp. 4204-4214, Nov. 2005.

[8] S. Singh, M. Woo, and C. S. Raghavendra, "Power-aware routing in mobile ad hoc networks," in Proc. ACM/IEEE MOBICOM, Oct. 1998, vol. 1, pp. 350-355.

[9] S. Park and M. Srivastava, "Power aware routing in sensor networks using dynamic source routing," ACM MONET Special Issue on Energy Conserving Protocols in Wireless Networks, 1999.

[10] R. C. Shah and J. Rabaey, "Energy aware routing for low energy ad hoc sensor networks," in Proc. IEEE WCNC, Mar. 2002, vol. 1, pp. 350-355.

[11] W. B. Heinzelman, A. P. Chandrakasan, and H. Balakrishnan, "An application specific protocol architecture for wireless microsensor networks," IEEE Trans. Wireless Commun., vol. 1, no. 4, pp. 660-670, Oct. 2002.

[12] A. Subramanian and A. H. Sayed, "A probablistic power-aware routing algorithm for wireless ad-hoc networks," in Proc. IEEE Veh. Technol. Conf. (VTC), May 2004, vol. 4, pp. 2190-2194.

[13] , "Performance analysis of a class of clustered wireless networks," in Proc. IEEE Workshop Signal Processing Advances Wireless Commun. (SPAWC), Lisbon, Portugal, July 2004, pp. 11-15.

[14] B. Bollobas, "Sharp concentration of measure phenomena in the theory of random graphs," in Random Graphs, M. Karonski, J. Jaworski, and A. Rucinski, Eds. New York: Wiley, 1990

[15] M. Loeve, Probability Theory. New York: Springer-Verlag, 1977, vol. 1.

[16] P. Gupta and P. R. Kumar, "Critical power for asymptotic connectivity in wireless networks," in Stochastic Analysis, Control, Optimization, and Applications: A Volume in Honor of W. H. Fleming, W. M. McEneay, G. Yin, and Q. Zhang, Eds. Boston: Birkhauser, 1998, pp. 547-566.

[17] A. Papoulis and S. U. Pillai, Probability, Random Variables, and Stochastic Processes. New York: McGraw-Hill, 2001.

[18] A. H. Sayed, Fundamentals of Adaptive Filtering. New York: John Wiley \& Sons, 2003.

[19] M. Grossglauser and D. Tse, "Mobility increseas the capacity of ad-hoc wireless networks," IEEE/ACM Trans. Networking, vol. 10, no. 4, pp. 477-486, Aug. 2002.

[20] P. Hall, Introduction to the Theory of Coverage Processes. New York: John Wiley and Sons, 1988.

[21] R. Motwani and P. Raghavan, Randomized Algorithms. Cambridge, UK: Cambridge University Press, 1995.

[22] N. Bambos, S. C. Chen, and G. J. Pottie, "Channel access algorithms with active link protection for wireless communication networks with power control," IEEE/ACM Trans. Networking, vol. 8, no. 5, pp. 583597, Oct. 2000.

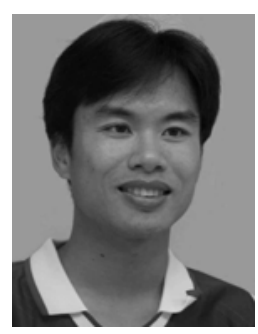

Zhi Quan received his B.E. degree in Communication Engineering from Beijing University of Posts and Telecommunications (BUPT), Beijing, China, in 1999, and his M.S. degree in Electrical Engineering from Oklahoma State University (OSU) in 2004. He is currently pursuing the Ph.D degree in electrical engineering at the University of California, Los Angeles (UCLA). His current research interests include statistic signal processing, wireless communications, and cognitive radio.

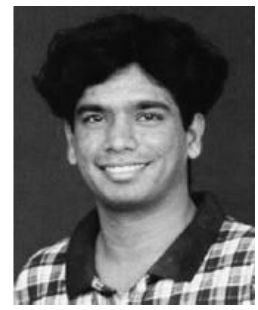

Ananth Subramanian (M'04) received the bachelor's degree in electrical and electronics engineering and master's degree in mathematics in 1997 from BITS, Pilani, India. He received the master's degree in electrical engineering from the Indian Institute of Science, Bangalore, in 1999 and the Ph.D. degree in electrical engineering in 2004 from the University of California, Los Angeles.

$\mathrm{He}$ is currently a Research Fellow at the Institute for Infocomm Research, A-STAR, Singapore. He has held the position of Teaching Fellow at UCLA. His areas of interest include statistical and robust signal processing, wireless communications, and wireless sensor and ad hoc networks. Dr. Subramanian was awarded the Gold Medal for being the best overall graduate student from the Indian Institute of Science and received the Henry Samueli Excellence in Teaching Award in 2002. He was also awarded the UCLA Chancellors Fellowship in 2003

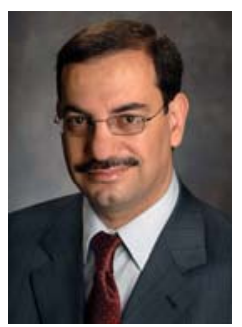

Ali H. Sayed (S'90-M'92-SM'99-F'01) received the $\mathrm{Ph} . \mathrm{D}$. degree in electrical engineering from Stanford University, Stanford, CA, in 1992, and the Ph.D. degree in electrical engineering from Stanford University, Stanford, CA, in 1992.

$\mathrm{He}$ is a Professor and the Chairman of the Electrical Engineering Department at the University of California, Los Angeles (UCLA). He is also the Principal Investigator of the UCLA Adaptive Systems Laboratory (www.ee.ucla.edu/asl). He is the author or coauthor of over 280 journals and conference publications and is the author of the textbook Fundamentals of Adaptive Filtering (New York: Wiley, 2003), the coauthor of the research monograph Indefinite Quadratic Estimation and Control (Philadelphia, PA: SIAM, 1999), and of the graduate-level textbook Linear Estimation (Englewood Cliffs, NJ: Prentice-Hall, 2000). He is also coeditor of Fast Reliable Algorithms for Matrices with Structure (Philadelphia, PA: SIAM 1999). He has contributed several articles to engineering and mathematical encyclopedias and handbooks and has served on the program committees of several international meetings. His research interests include adaptive and statistical signal processing, distributed processing, filtering and estimation theories, signal processing for communications, interplays between signal processing and control methodologies, system theory, and fast algorithms for large-scale problems.

Dr. Sayed is a recipient of the 1996 IEEE Donald G. Fink Award, a 2002 Best Paper Award from the IEEE Signal Processing Society, the 2003 Kuwait Prize in Basic Science, the 2005 Frederick E. Terman Award, the 2005 Young Author Best Paper Award from the IEEE Signal Processing Society, and two Best Student Paper awards at international meetings. He is also a member of the technical committees on Signal Processing Theory and Methods (SPTM) and on Signal Processing for Communications (SPCOM), both of the IEEE Signal Processing Society. He is a member of the editorial board of IEEE Signal Processing Magazine. He has served as Editor-in-Chief of the IEEE TRANSACTIONS ON SignAl PROCESSING (2003-2005) and is now serving as Editor-in-Chief of the EURASIP Journal on Advances in Signal Processing. $\mathrm{He}$ is General Chairman of the International Conference on Acoustics, Speech, and Signal Processing (ICASSP) 2008, and is a member of the Board of Governors of the IEEE Signal Processing Society. 